\title{
Complex post-traumatic stress disorder: a new diagnosis in ICD $-11^{\dagger}$
}

\author{
Chris R. Brewin (1)
}

\begin{abstract}
SUMMARY
The World Health Organization's proposals in ICD-11, released for comment by member states in 2018, introduce for the first time in a major diagnostic system a distinction between post-traumatic stress disorder (PTSD) and complex post-traumatic stress disorder (CPTSD). This article sets the new diagnosis of CPTSD within the context of previous similar formulations, describes its definition and requirements, and reviews the evidence concerning its epidemiology, differential diagnosis, assessment and treatment.
\end{abstract}

\section{LEARNING OBJECTIVES}

After reading this article you will be able to:

- understand the relationship between CPTSD and precipitating events

- distinguish CPTSD from PTSD and borderline personality disorder

- recognise current issues and practices in psychological treatment.

\section{DECLARATION OF INTEREST}

C.B. was an unpaid member of the Working Group on Classification of Stress-Related Disorders for the World Health Organization's International Advisory Group for the Revision of ICD-10 Mental and Behavioural Disorders. The views expressed in this article are those of the author and do not represent the official policies or positions of the International Advisory Group or the WHO.

\section{KEYWORDS}

Post-traumatic stress disorder; cognitive-behavioural therapies; trauma.

In July 2018 the World Health Organization (WHO) formally issued ICD-11, the latest revision of the International Classification of Diseases, for consultation with member states (World Health Organization 2018). It contains major changes to the diagnosis of post-traumatic stress disorder (PTSD), which is now replaced by two diagnoses, PTSD and complex PTSD (CPTSD) (Maercker 2013). Consistent with the ICD-11 principle that diagnoses should be simple and have the maximum clinical utility, requirements for PTSD include evidence of the re-experiencing of traumatic events in the present, deliberate avoidance, a current sense of threat and functional impairment. CPTSD requires the same but, in addition, evidence of disturbances in self-organisation (DSO), consisting of affect dysregulation, negative self-concept and disturbances in relationships. A more detailed rationale for the divergence from PTSD as defined in ICD-10, DSM-IV and DSM-5 has been presented elsewhere (Brewin 2013, 2017).

The term 'complex PTSD' was first used to describe a syndrome experienced by survivors of repeated, prolonged trauma and involving alterations in affect regulation, consciousness, self-perception and relationships to the perpetrator and to others (Herman 1992). Other similar diagnoses have previously been put forward. One is the ICD10 diagnosis 'enduring personality change after catastrophic experience' (EPCACE), which described the disturbances in self-organisation that can sometimes result from multiple, chronic or repeated traumas from which escape is difficult or impossible (e.g. childhood abuse, domestic violence, torture, war, imprisonment) (World Health Organization 1992). Another is 'disorders of extreme stress not otherwise specified' (DESNOS), which was included in the Appendix to DSM-IV (American Psychiatric Association 1994). The DESNOS diagnosis has been operationalised using 48 possible symptoms, organised into 6 scales and 27 subscales (Pelcovitz 1997). A comparable diagnosis for children is developmental trauma disorder (DTD) (Ford 2018).

A number of practical difficulties have been identified with these earlier formulations (Resick 2012). These include the large number of candidate symptoms and the substantial symptom overlap with other diagnoses, such as DSM-IV PTSD, major depression and borderline personality disorder (American Psychiatric Association 1994). Evidence has also been lacking that these formulations are uniquely linked to chronic or repeated trauma. There has been uncertainty over whether these formulations represent a complex and severe form of PTSD or a syndrome distinct from (although often comorbid with) PTSD (Ford 1999). Relatedly, it has been pointed out that complex PTSD might simply represent one end of a spectrum of severity
Chris R. Brewin, FAcSS, FMedSci, FBA, is Emeritus Professor of Clinical Psychology at University College London and a former consultant clinical psychologist at the Traumatic Stress Clinic, part of Camden \& Islington NHS Foundation Trust, London, UK.

Correspondence Professor Chris R. Brewin, Department of Clinical, Educational and Health Psychology, University College London, Gower Street, London WC1E 6BT, UK. Email: c.brewin@ucl.ac.uk

First received 22 Mar 2019 Final revision 21 Jun 2019 Accepted 28 Jun 2019

Copyright and usage (C) The Royal College of Psychiatrists 2019

See commentary, this issue. 


\section{B0X 1 ICD-11 CPTSD definition and diagnostic guidelines}

\section{Definition}

Complex post-traumatic stress disorder (complex PTSD) is a disorder that may develop following exposure to an event or series of events of an extremely threatening or horrific nature, most commonly prolonged or repetitive events from which escape is difficult or impossible (e.g., torture, slavery, genocide campaigns, prolonged domestic violence, repeated childhood sexual or physical abuse). All diagnostic requirements for PTSD are met. In addition, Complex PTSD is characterized by severe and persistent 1) problems in affect regulation; 2) beliefs about oneself as diminished, defeated or worthless, accompanied by feelings of shame, guilt or failure related to the traumatic event; and 3) difficulties in sustaining relationships and in feeling close to others. These symptoms cause significant impairment in personal, family, social, educational, occupational or other important areas of functioning.

Diagnostic guidelines

- Exposure to a stressor typically of an extreme or prolonged nature and from which escape is difficult or impossible such as torture, concentration camps, slavery, genocide campaigns and other forms of organized violence, domestic violence, and childhood sexual or physical abuse.

- Presence of the core symptoms of PTSD (re-experiencing the trauma in the present, avoidance of reminders of the trauma, and persistent perceptions of current threat).

- Following onset of the stressor event and co-occurring with PTSD symptoms, there is the development of persistent and pervasive impairments in affective, self and relational functioning including problems in affect regulation, persistent beliefs about oneself as diminished, defeated or worthless, persistent difficulties in sustaining relationships.

The stressors associated with Complex PTSD are typically of an interpersonal nature, that is are the result of human mistreatment rather than acts of nature (e.g., earthquakes, tornadoes, tsunamis) or accidents (train wrecks, motor vehicle accidents). In addition to the typical symptoms of PTSD, Complex PTSD is characterized by more persistent long-term problems in affective, self and relational functioning. Problems in all three areas are often co-occurring

(Bisson 2019) from being recognised as a diagnosis in its own right. First, ICD-11 clearly positions CPTSD as a separate diagnosis from PTSD (a person can be diagnosed with PTSD or CPTSD but not both). At the same time, CPTSD must include the same evidence for re-experiencing in the present, avoidance and sense of threat that is part of PTSD. Thus, it shares with PTSD an explicit focus on specific, identifiable traumatic events that are prominent in consciousness, rather than being a non-specific response to extreme trauma. Finally, chronic or repeated trauma is a risk factor, not a requirement, for CPTSD. There is an acknowledgment that there is no necessary connection with severe trauma, meaning that it can be diagnosed after a single traumatic event (although this will be less likely).

\section{Epidemiology}

One of the first studies using the new ICD-11 diagnosis in a nationally representative sample of traumaexposed adults was conducted in Israel, finding a 1-month prevalence of $9 \%$ for ICD-11 PTSD and $2.6 \%$ for CPTSD. Women reported a higher rate of PTSD than men but did not differ in the CPTSD rate (Ben-Ezra 2018). A nationwide sample in Germany, in contrast, found a 1-month prevalence of $1.5 \%$ for PTSD and $0.5 \%$ for CPTSD, with no significant gender differences (Maercker 2018). A nationally representative survey in the USA has reported total rates for both diagnoses in between these studies, with PTSD at $3.4 \%$ and CPTSD at 3.5\% (Cloitre 2019); women reported higher rates of both PTSD and CPTSD.

The requirements for PTSD in ICD-10 were less specific than in ICD-11 and did not include evidence of functional impairment. In DSM-5 the PTSD diagnosis is broader, in that it includes many of the symptoms that belong to both ICD-11 PTSD and CPTSD. A substantial number of investigators have now compared rates within the same community or treatment-seeking samples of ICD-10 PTSD, ICD-11 PTSD/CPTSD combined, and PTSD diagnosed using DSM-IV or DSM-5. A review of these studies found that in adult samples the combined ICD-11 PTSD/CPTSD rate is reliably lower than both the ICD-10 and DSMIV/DSM-5 rates, consistent with the wish to define the disorder more narrowly (Brewin 2017).

At present there is only very preliminary evidence to support the existence of CPTSD in children and adolescents, according to a position paper published by the International Society for Traumatic Stress Studies (ISTSS Guidelines Committee 2019). In community samples, however, the few studies available do not suggest any difference in prevalence
This greater definitional clarity resolves a number of the problems that have hindered 'complex PTSD' 
rates among children and young people between ICD-11 PTSD/CPTSD combined and DSM-IV/ DSM-5 PTSD (Brewin 2017).

\section{Differential diagnosis}

A number of studies have conducted latent profile analysis or latent class analysis to test the assumption that there are different clinical groups corresponding to PTSD and CPTSD. With an occasional exception, these have consistently found one group of patients who report re-experiencing in the present, avoidance and sense of threat, and another group who report elevated levels of these same symptoms but in addition report problems in affect regulation, social relationships and a disturbed sense of self (Brewin 2017). Similar findings have been reported for children and adolescents. Factor analytic studies have also consistently found evidence for six clusters of symptoms, with three related to a PTSD higher-order factor and three to a DSO higher-order factor in the expected way (Brewin 2017). The cross-cultural validity of the proposals have been tested in a number of countries, including Austria, Denmark, Germany, Israel and Lithuania, and the distinction also appears to be applicable to samples of refugees (Vallières 2018; Frost 2019).

Consistent with the idea that chronic or multiple trauma is a risk factor for CPTSD, studies have shown that childhood physical or sexual abuse, particularly within the family, is more strongly related to CPTSD than PTSD (Cloitre 2019). CPTSD is also associated with higher levels of psychiatric burden than PTSD, including greater depression and dissociation (Hyland 2018; Cloitre 2019).

Questions have been raised about the potential overlap between CPTSD and other disorders for which prolonged or repeated trauma is thought to be a risk factor, such as borderline personality disorder (BPD). Research is in its early stages but two studies employing latent class analysis on samples reporting a history of childhood abuse (Cloitre 2014; Frost 2018) and one study that used network analysis on an institutionally abused sample (Knefel 2016) have suggested that the two disorders can be meaningfully distinguished. The first point to note is that trauma exposure and PTSD symptoms are required for a CPTSD diagnosis but not a BPD diagnosis. Further, the symptoms that are more characteristic of BPD than of CPTSD are being frantic about being abandoned, having an unstable sense of self, having unstable relationships, impulsiveness, and self-harm and suicidal behaviour. The symptoms that are more characteristic of CPTSD than of BPD are an extremely negative sense of self and avoidance of relationships with no significant shifts in identity.
Some individuals with a history of prolonged or repeated trauma may present with comorbid BPD and CPTSD. In such people the utility of the CPTSD diagnosis is primarily to identify active trauma symptoms that are affecting mental state and behaviour, whereas the utility of the BPD diagnosis is to identify that safety considerations are prominent and are likely to become more so if trauma symptoms are confronted directly without proper preparation.

\section{Assessment of CPTSD}

ICD-11 PTSD and CPTSD are more tightly defined diagnoses than DSM-5 PTSD, requiring a smaller number of more specific symptoms to be diagnosed. For example, DSM-5 includes five re-experiencing symptoms involving any kind of intrusive memory, as well as any kind of emotional or physiological reaction on encountering reminders of the event (American Psychiatric Association 2013). Because several of these symptoms, including intrusive memories, are found in many other psychiatric disorders, ICD-11 requires that there should be an element of re-experiencing in the present. This involves one of two symptoms: either a nightmare that recapitulates some aspect of the event (but does not have to be an exact replay) or a daytime flashback in which the event is vividly replayed. DSM-5 and ICD-11 now both define flashbacks as existing on a continuum: at one end is total absorption in the traumatic memory, with a complete loss of awareness of the current environment, and at the other is a vivid intrusive memory of the traumatic event in which the person does not lose contact with their current surroundings but has a sense, however fleeting, that the event is happening again in the here and now. This requirement is important to differentiate PTSD from other conditions - such as major depression - in which people have intrusive memories of distressing events but experience them as belonging to the past. If the person has no conscious memory of the event (perhaps because of a head injury or intoxication) ICD-11 allows this criterion to be met by an emotional response to reminders of it.

ICD-11 also requires evidence (again, at least one symptom) of both avoidance and a sense of threat. Although the deliberate avoidance symptoms are the same as in DSM-5, the hyperarousal symptom cluster is replaced in ICD-11 by the more specific construct of a continuing sense of threat despite the event being in the past. This can be manifested either by hypervigilance or an exaggerated startle reaction. The requirement for functional impairment in some important aspect of the person's life also differentiates PTSD in ICD-11 from the equivalent diagnosis in ICD-10. 
For CPTSD to be diagnosed the person must also demonstrate pervasive, long-standing disturbances in self-organisation (DSO). DSO consist of three components, and again there must be evidence, in the form of at least one symptom, that all three are simultaneously present. Affective dysregulation may take the form of hyperactivation, the tendency to experience intense emotions that cannot readily be moderated, or of hypoactivation, in which there is an absence of normal feeling states, or of both. Negative self-concept refers to feelings of worthlessness or being a failure, while the 'disturbances in relationships' component focuses on detachment and withdrawal from others. These symptoms too must be accompanied by evidence of impairment in important life roles.

Different aspects of CPTSD may be more salient than others, depending on the type of stressor. For example, uncontrollable anger tends to have relatively low endorsement as part of the affect dysregulation cluster among adults with childhood sexual and/or physical abuse (Cloitre 2014), but much higher endorsement among those who were exposed to armed conflict and abducted into child soldiering (Murphy 2016). ICD-11 further recognises that many other symptoms (Box 2) commonly accompany a PTSD presentation, even though they do not discriminate it from other disorders.

Although there are a few instruments that assess different forms of complex PTSD (Pelcovitz 1997; Litvin 2017), at present only one instrument is available that specifically assesses ICD-11 CPTSD, the International Trauma Questionnaire (Cloitre 2018). The questionnaire is in use in at least 29 countries on six continents. A companion structured interview measure is under development.

\section{BOX 2 Symptoms commonly accompanying ICD-11 PTSD}

- Anxiety symptoms such as panic, obsessions and compulsions

- Ruminative thoughts indicating preoccupation with the traumatic event(s)

- General dysphoria in the form of emotional blunting, anhedonia, lack of a perceived future, insomnia, irritability and concentration problems

- Dissociative symptoms such as memory disturbances (e.g. dissociative amnesia) and pseudohallucinations (e.g. hearing own thoughts as voices)

- Suicidal ideation and behaviour

- Changes in interpersonal attitudes and behaviour, such as social withdrawal, suspicion and distrust

- Excessive use of alcohol or drugs to avoid re-experiencing

- Excessive risk-taking (e.g. dangerous driving)

- Psychotic reactions with hallucinations and delusions related to the trauma

(Bisson 2019)

- Somatic complaints without organic basis, such as headache and dyspnoea

\section{Treatment of complex PTSD}

An important debate has been taking place over whether complex PTSD requires a different kind of psychological treatment to PTSD. The International Society for Traumatic Stress Studies produced a position paper (ISTSS Guidelines Committee 2019) recommending three stages or phases of treatment, each with a distinct function. Phase 1 focuses on ensuring the individual's safety, reducing symptoms, and increasing important emotional, social and psychological competencies. Phase 2 focuses on processing the unresolved aspects of the individual's memories of traumatic experiences so that these are integrated into an adaptive representation of self, relationships and the world, using standard or slightly adapted methods taken from conventional trauma-focused cognitive-behavioural therapy. Phase 3 involves consolidation of treatment gains to facilitate the transition from treatment into a greater engagement with the outside world.

Currently validated treatments include Skills Training for Affective and Interpersonal Regulation combined with Modified Prolonged Exposure (STAIR/MPE) (Cloitre 2010), which includes a first phase emphasising the acquisition of affective and interpersonal regulation skills followed by a modified version of prolonged exposure to address the traumatic memories. Another alternative that could be considered for Phase 1 is compassion-focused therapy (Karatzias 2019a). This addresses the frequent tendency for those with CPTSD to feel intense shame and to be highly selfblaming and self-denigratory, reactions that are risk factors for PTSD and would be expected to make exposure to the traumatic memories too painful to tolerate. Treatment of children and adolescents with a phase-based approach has been found to achieve similar gains in those diagnosed with CPTSD as in those with PTSD, although those with CPTSD started and finished therapy with higher symptom levels (Sachser 2017).

However, the need for a phased approach to treatment has been challenged by other experts (de Jongh 2016). They pointed to the lack of direct evidence for the superiority of a phase-based approach, as well numerous indications that immediate traumafocused treatment (i.e. bypassing phase 1) could be effective for many patients with histories of multiple traumatisation, including childhood abuse. For example, an intensive form of trauma-focused treatment that involved twelve 90-minute sessions of prolonged exposure over 4 days, followed by four weekly 90-minute booster sessions, achieved large treatment effects that persisted over 6 months (Hendriks 2018). A recent meta-analysis 
(Karatzias 2019b) has confirmed that standard treatments for PTSD do reduce CPTSD symptoms of negative self-concept and disturbances in relationships, although little evidence is available for affective dysregulation. The analysis also found that treatment gains were reduced when trauma exposure dated from childhood.

This debate largely preceded the current conceptualisation of CPTSD in ICD-11, which has refocused attention on the presenting symptoms rather than the nature of the trauma. Rigorous comparisons of alternative treatment approaches are lacking but it is unlikely that diagnostic concerns alone will prove to be decisive. Theoretically, the factors that are thought to undermine direct work with trauma memories generally involve cognitive and emotional reactions that prevent the person from holding the most traumatic material in consciousness and keeping a degree of detachment and reflection as they do so (Brewin 2010a). For example, loss of trust very frequently accompanies CPTSD (Ebert 2004) and may impede the formation of a therapeutic relationship strong enough to allow the patient to share critical experiences or even revisit them privately. This is to be expected, given that trauma survivors not infrequently have the experience of being disbelieved or denigrated, or are betrayed by individuals or organisations who have a duty of care towards them. Some other factors that affect CPTSD treatment are discussed further below.

What is important is that, for some patients, a stabilisation phase prior to trauma-focused treatment

BOX 3 NICE recommendations for traumafocused cognitive-behavioural interventions for CPTSD

- Build in extra time to develop trust with the person, by increasing the duration or the number of therapy sessions according to the person's needs

- Take into account the safety and stability of the person's personal circumstances (e.g. their housing situation) and how this might affect engagement with and success of treatment

- Help the person manage any problems that might be a barrier to engaging with trauma-focused therapies, such as substance misuse, dissociation, emotional dysregulation, interpersonal difficulties or negative selfperception

- Work with the person to plan any ongoing support they will need after the end of treatment, for example to manage any residual PTSD symptoms or comorbid psychiatric conditions

(National Institute for Health and Care Excellence 2018) that directly addresses their traumatic memories will strengthen the therapeutic relationship and prevent drop-out. Consistent with this, the recently updated NICE guideline on PTSD (National Institute for Health and Care Excellence 2018) notes that trauma-focused cognitive-behavioural interventions should normally be provided over 8-12 sessions but may need to be extended for those with more complex presentations. Specific recommendations for such presentations are listed in Box 3. NICE does not give any indication of how much additional time might be needed, but therapeutic experience indicates that, although 20-30 sessions will be sufficient for many, 1-2 years of weekly therapy may be needed for the more complex cases.

\section{Factors affecting the treatment of CPTSD}

There are numerous clinical challenges that are regularly encountered in the management of CPTSD and that interfere with psychological treatment even in patients who are well-motivated and engaged. Of these, chronic dissociation and/or voice-hearing are among the most common.

\section{Dissociation}

The tendency for patients to dissociate during therapy sessions when confronted by traumatic reminders is well recognised. Dissociation can involve either too much absorption in or too much disengagement from the traumatic material. In either case the ability to reflect deliberately on the material, essential for positive therapeutic change, may be compromised. However, the literature shows that dissociative symptoms tend to improve with PTSD treatment and need not be a barrier to a good outcome. The outcome is likely to depend on how successfully dissociation can be managed in the individual person.

CPTSD, however, may be accompanied by much more pervasive dissociation, including complete loss of awareness of the current environment (sometimes in the form of a fugue state) that occurs both in the therapy session and in everyday situations such as crossing roads. Such episodes are usually frightening and potentially put the patient at increased risk - it is likely that they will need to be addressed before the commencement of direct trauma work that might exacerbate them. The presence of these episodes can be assessed using the Dissociative Experiences Scale (Carlson 1993) or a briefer 10-item version that focuses on the most pathological dissociation symptoms (Waller 1996). An adolescent version is also available (Armstrong 1997). 
MCO answers

$1 c \quad 2 d \quad 3$ e 4 b 5 c
Stabilisation work may therefore be required to assess which external situations provoke such reactions and to teach the patient to monitor and control them, for example using grounding techniques (Kennedy 2013). In vivo practice accompanied by a therapist may be required occasionally in order to guarantee the patient's safety in real-world roles such as driver or pedestrian. Severe dissociative reactions occurring in the therapeutic session are also likely to be frightening and may require the traumatic memories to be approached very slowly and gradually, greatly extending the therapeutic process.

\section{Voice-hearing}

Although now recognised as an associated feature of PTSD in both DSM-5 and ICD-11, the symptom whereby patients report hearing their thoughts in the form of a voice speaking to them is rarely acknowledged in textbooks or treatment manuals. Following a number of observational studies of PTSD in the US military, voice-hearing has been identified as prevalent in UK military and civilian samples, particularly in those with more complex forms of the disorder (Anketell 2010; Brewin 2010b). These studies reported voice-hearing to be correlated with increased dissociative symptoms, consistent with some theoretical views of voicehearing in people with psychosis (McCarthy-Jones 2015). Although the average number of different voices is generally between one and three, the presence of a large number of voices indicates that the person may attract a comorbid diagnosis of a dissociative disorder.

Clinical experience confirms that voice-hearing often has a very substantial impact on the lives of people with CPTSD, with the potential to greatly worsen mood and alter their sense of identity. Further, voices may be active in the therapeutic environment, commenting on mental health professionals and their interventions and sometimes counselling non-cooperation. Voices appear to have greater impact than negative thoughts because, similar to the experience in psychosis, patients describe relationships with them in which the patient often feels inadequate or intimidated (Brewin 2010b).

Techniques proposed for working psychologically with voices in people with psychosis (Corstens 2012) are relevant to people with CPTSD (Brewin 2019). It appears to be helpful to explore the individual's attitudes to and assumptions about their voices, as well as the content, with the aim of destigmatising the experience of voice-hearing and reassuring the person about their sanity. It is likely that the presence of voices will not previously have been disclosed to anyone. At the same time individuals can be taught to question and evaluate the content of what the voices say, using standard techniques of Socratic questioning that are part of cognitive therapy. These methods often enable the person to distance themselves from their voices for the first time, stop treating them as infallible and accept them as a part of their mental life that needs to be acknowledged rather than believed or obeyed.

\section{Conclusions}

Complex PTSD has been discussed in one form or another for many years but now, in ICD-11, the condition has been defined in a way that is consistent with empirical evidence that it is not inevitably linked to certain types of traumatic exposure. The presence of re-experiencing, avoidance and 'sense of threat' symptoms also helps to demarcate it from other disorders that may be the result of prolonged or repeated trauma. In its new form, CPTSD can be readily distinguished by clinicians (as established in an ICD-11 field study by Keeley 2016) and meets a long-expressed need. It also, as discussed by NICE (2018), has resource implications, because brief treatments are unlikely to be adequate. Although conventional trauma-focused treatment may be effective for some, there are numerous complicating factors that will require practitioners specialising in CPTSD to develop additional skill sets.

\section{References}

American Psychiatric Association (1994) Diagnostic and Statistical Manual of Mental Disorders (4th edn) (DSM-IV). APA.

American Psychiatric Association (1994) Diagnostic and Statistical Manual of Mental Disorders (4th edn) (DSM-IV). APA.

American Psychiatric Association (2013) Diagnostic and Statistical Manual of Mental Disorders (5th edn) (DSM-5). American Psychiatric Publishing.

Anketell C, Dorahy MJ, Shannon M, et al (2010) An exploratory analysis of voice hearing in chronic PTSD: potential associated mechanisms. Journal of Trauma \& Dissociation, 11: 93-107.

Armstrong JG, Putnam FW, Carlson EB, et al (1997) Development and validation of a measure of adolescent dissociation: the Adolescent Dissociative Experiences Scale. Journal of Nervous and Mental Disease, 185: 491-7.

Ben-Ezra M, Karatzias T, Hyland P, et al (2018) Posttraumatic stress disorder (PTSD) and complex PTSD (CPTSD) as per ICD-11 proposals: a population study in Israel. Depression and Anxiety, 35: 264-74.

Bisson Jl, Brewin CR, Cloitre M, Maercker A (2019) PTSD and Complex PTSD: Diagnosis, assessment, and screening. In Effective treatments for PTSD (3rd edn) (eds EB Foa, TM Keane, MJ Friedman, JA Cohen). Guilford.

Brewin CR, Gregory JD, Lipton M, et al (2010a) Intrusive images in psychological disorders: characteristics, neural mechanisms, and treatment implications. Psychological Review, 117: 210-32.

Brewin CR, Patel T (2010b) Auditory pseudo-hallucinations in United Kingdom war veterans and civilians with posttraumatic stress disorder. Journal of Clinical Psychiatry, 71: 419-25. 
Brewin CR (2013) 'I wouldn't start from here' - an alternative perspective on PTSD from the ICD-11: comment on Friedman (2013). Journal of Traumatic Stress, 26: 557-9.

Brewin CR, Cloitre M, Hyland P, et al (2017) A review of current evidence regarding the ICD-11 proposals for diagnosing PTSD and complex PTSD. Clinical Psychology Review, 58: 1-15

Brewin CR (2019) Treating voice-hearing in PTSD. Advances in Cognitive Therapy, 20: 3-.

Carlson EB, Putnam FW (1993) An update on the Dissociative Experiences Scale. Dissociation: Progress in the Dissociative Disorders, 6: 16-27.

Cloitre M, Stovall-McClough KC, Nooner K, et al (2010) Treatment for PTSD related to childhood abuse: a randomized controlled trial. American Journal of Psychiatry, 167: 915-24.

Cloitre M, Garvert DW, Weiss B, et al (2014) Distinguishing PTSD, complex PTSD, and borderline personality disorder: a latent class analysis. European Journal of Psychotraumatology, 5: doi 10.3402/ejpt. v5.25097.

Cloitre M, Shevlin M, Brewin CR, et al (2018) The International Trauma Questionnaire: development of a self-report measure of ICD-11 PTSD and complex PTSD. Acta Psychiatrica Scandinavica, 138: 536-46.

Cloitre M, Hyland P, Bisson Jl, et al (2019) ICD-11 PTSD and complex PTSD in the United States: a population-based study. Journal of Traumatic Stress, in press.

Corstens D, Longden E, May R (2012) Talking with voices: exploring what is expressed by the voices people hear. Psychosis: Psychological, Social and Integrative Approaches, 4: 95-104

de Jongh A, Resick PA, Zoellner LA, et al (2016) Critical analysis of the current treatment guidelines for complex PTSD in adults. Depression and Anxiety, 33: 359-69.

Ebert A, Dyck MJ (2004) The experience of mental death: the core feature of complex posttraumatic stress disorder. Clinical Psychology Review, 24: 617-35

Ford JD (1999) Disorders of extreme stress following war-zone military trauma: Associated features of posttraumatic stress disorder or comorbid but distinct syndromes? Journal of Consulting and Clinical Psychology, 67 : $3-12$

Ford JD, Spinazzola J, van der Kolk B, et al (2018) Toward an empirically based developmental trauma disorder diagnosis for children: factor structure, item characteristics, reliability, and validity of the Developmental Trauma Disorder semi-structured interview. Journal of Clinical Psychiatry, 79(5): $17 \mathrm{~m} 11675$.

Frost R, Hyland P, Shevlin M et al (2018) Distinguishing Complex PTSD from Borderline Personality Disorder among individuals with a history of sexual trauma: a latent class analysis. European Journal of Trauma and Dissociation, doi 10.1016/j.ejtd.2018.08.004

Frost R, Hyland P, McCarthy A, et al (2019) The complexity of trauma exposure and response: profiling PTSD and CPTSD among a refugee sample. Psychological Trauma: Theory, Research, Practice and Policy, 11: $165-75$.

Hendriks L, de Kleine RA, Broekman TG, et al (2018) Intensive prolonged exposure therapy for chronic PTSD patients following multiple trauma and multiple treatment attempts. European Journal of Psychotraumatology, 9 (1): 1425574

Herman JL (1992) Complex PTSD - a syndrome in survivors of prolonged and repeated trauma. Journal of Traumatic Stress, 5: 377-91.

Hyland P, Shevlin M, Fyvie C, et al (2018) Posttraumatic stress disorder and complex posttraumatic stress disorder in DSM-5 and ICD-11: clinical and behavioral correlates. Journal of Traumatic Stress, 31: $174-80$.

ISTSS Guidelines Committee (2019) ISTSS Guidelines Position Paper on Complex PTSD in Adults. ISTSS (http://www.istss.org/getattachment/
Treating-Trauma/New-ISTSS-Prevention-and-Treatment-Guidelines/ ISTSS_CPTSD-Position-Paper-(Adults)_FNL.pdf.aspx).

Karatzias T, Hyland P, Bradley A, et al (2019a) Is self-compassion a worthwhile therapeutic target for ICD-11 complex PTSD (CPTSD)? Behavioural and Cognitive Psychotherapy, 47: 257-69.

Karatzias T, Murphy P, Cloitre M, et al (2019b) Psychological interventions for ICD-11 complex PTSD symptoms: systematic review and meta-analysis. Psychological Medicine, Mar 12: doi 0.1017/S0033291719000436 [Epub ahead of print].

Keeley JW, Reed GM, Roberts MC, et al (2016) Disorders specifically associated with stress: a case-controlled field study for ICD-11 mental and behavioural disorders. International Journal of Clinical and Health Psychology, 16: 109-27.

Kennedy F, Kennerley H, Pearson D (eds) (2013) Cognitive Behavioural Approaches to the Understanding and Treatment of Dissociation. Routledge.

Knefel M, Tran US, Lueger-Schuster B (2016) The association of posttraumatic stress disorder, complex posttraumatic stress disorder, and borderline personality disorder from a network analytical perspective. Journal of Anxiety Disorders, 43: 70-8.

Litvin JM, Kaminski PL, Riggs SA (2017) The Complex Trauma Inventory: a self-report measure of posttraumatic stress disorder and complex posttraumatic stress disorder. Journal of Traumatic Stress, 30: 602-13.

Maercker A, Brewin CR, Bryant RA, et al (2013) Diagnosis and classification of disorders specifically associated with stress: proposals for ICD-11. World Psychiatry, 12: 198-206.

Maercker A, Hecker T, Augsburger M, et al (2018) ICD-11 prevalence rates of posttraumatic stress disorder and complex posttraumtic stress disorder in a German nationwide sample. Journal of Nervous and Mental Disease, 206: 270-6.

McCarthy-Jones S, Longden E (2015) Auditory verbal hallucinations in schizophrenia and post-traumatic stress disorder: common phenomenology, common cause, common interventions? Frontiers in Psychology, 6: 1071 (doi: 10.3389/fpsyg.2015.01071)

Murphy S, Elklit A, Dokkedahl S, et al (2016) Testing the validity of the proposed ICD-11 PTSD and complex PTSD criteria using a sample from Northern Uganda. European Journal of Psychotraumatology, 7: 32678.

National Institute for Health and Care Excellence (2018) Post-Traumatic Stress Disorder (NICE Guideline NG116). NICE.

Pelcovitz D, van der Kolk B, Roth S, et al (1997) Development of a criteria set and a structured interview for disorders of extreme stress (SIDES). Journal of Traumatic Stress, 10:3-16.

Resick PA, Bovin MJ, Calloway AL, et al (2012) A critical evaluation of the complex PTSD literature: implications for DSM-5. Journal of Traumatic Stress, 25: 241-51.

Sachser C, Keller F, Goldbeck L (2017) Complex PTSD as proposed for ICD11: validation of a new disorder in children and adolescents and their response to trauma-focused cognitive behavioral therapy. Journal of Child Psychology and Psychiatry, 58: 160-8.

Vallières F, Ceannt R, Daccache F, et al (2018) ICD-11 PTSD and complex PTSD amongst Syrian refugees in Lebanon: the factor structure and the clinical utility of the International Trauma Questionnaire. Acta Psychiatrica Scandinavica, 138: 547-57.

Waller NG, Putnam FW, Carlson EB (1996) Types of dissociation and dissociative types: a taxometric analysis of dissociative experiences. Psychological Methods, 1: 300-21.

World Health Organization (1992) The ICD-10 Classification of Mental and Behavioural Disorders: Clinical Descriptions and Diagnostic Guidelines. WHO

World Health Organization (2018) ICD-11: International Classification of Diseases 11th Revision. WHO (https://icd.who.int/en/). Accessed 22 Jul 2019. 


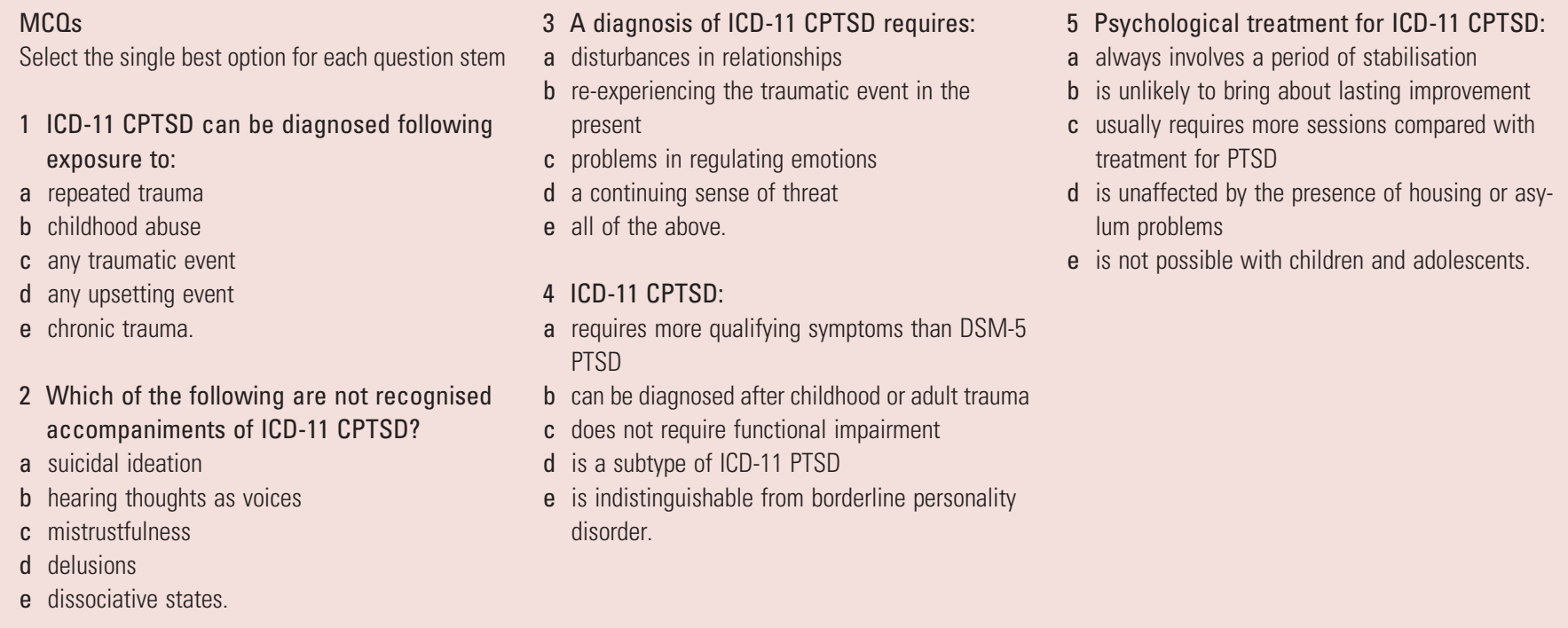

